\title{
Ocean wave spectrum estimation using measured vessel motions from an in-service
} container ship

Nielsen, Ulrik D.; Dietz, Jesper

Published in:

Marine Structures

Link to article, DOI:

10.1016/j.marstruc.2019.102682

Publication date:

2020

Document Version

Peer reviewed version

Link back to DTU Orbit

Citation (APA):

Nielsen, U. D., \& Dietz, J. (2020). Ocean wave spectrum estimation using measured vessel motions from an inservice container ship. Marine Structures, 69, [102682]. https://doi.org/10.1016/j.marstruc.2019.102682

\section{General rights}

Copyright and moral rights for the publications made accessible in the public portal are retained by the authors and/or other copyright owners and it is a condition of accessing publications that users recognise and abide by the legal requirements associated with these rights.

- Users may download and print one copy of any publication from the public portal for the purpose of private study or research.

- You may not further distribute the material or use it for any profit-making activity or commercial gain

- You may freely distribute the URL identifying the publication in the public portal

If you believe that this document breaches copyright please contact us providing details, and we will remove access to the work immediately and investigate your claim 


\title{
Ocean wave spectrum estimation using measured vessel motions from an in-service container ship
}

\author{
Ulrik D. Nielsen ${ }^{\mathrm{a}, \mathrm{c}}$, Jesper Dietz ${ }^{\mathrm{b}}$ \\ ${ }^{a}$ DTU Mechanical Engineering, Technical University of Denmark, DK-2800 Kgs. Lyngby, Denmark \\ ${ }^{b}$ Maersk Line, DK-1098 Copenhagen, Denmark \\ ${ }^{c}$ Centre for Autonomous Marine Operations and Systems, NTNU AMOS, NO-7491 Trondheim, Norway
}

\begin{abstract}
This article is about the use of measured wave-induced vessel motions for estimation of ocean wave spectra by application of the wave buoy analogy. In the study, data from a larger, in-service container ship is considered. The estimation of wave spectra, equivalently sea state parameters, is based on measurements from, respectively, a gyro and two accelerometers leading to the simultaneous use of the pitching motion together with the horizontal and vertical accelerations in a position close to the forward perpendicular. The study of in-service data leads to contemplations about the vessel's advance speed, as the possible existence of sea current means that speed-over-ground (SOG) and speed-through-water (STW) will be different. The article discusses aspects related to advance speed in the context of the wave buoy analogy, and a smaller sensitivity study is conducted. Preceding to the sensitivity study, a comparison is made between sea state estimates by the wave buoy analogy and estimates obtained from a hindcast study. The article shows an acceptable agreement between the two sets of estimates. Following, the main conclusion from the sensitivity study on advance speed is that errors and uncertainties in the speed log have an effect on the estimates of the wave buoy analogy. In fact, the effect can be severe if reliable STW measurements are not available. In the final part, the article includes a few discussions about (non)stationary conditions in the context of the wave buoy analogy, and, although the effect on results is not necessarily detrimental, care must be shown when the wave buoy analogy is applied during in-service conditions.
\end{abstract}

Keywords:

Wave spectrum estimation, ship motions, directional wave spectrum, in-service data, container ship, speed-through-water

\section{Introduction}

Knowledge about the sea state is valuable in all stages of marine operations; from the early design of the particular marine structure, to the actual operation of the structure, and finally in making post-voyage evaluations of the specific operation. Independent on the stage, attained knowledge of the sea state can benefit both fuel efficiency and safety of marine operations, which, for instance, could involve merchant ships' sailing in seaways, where motion control systems require the sea state as input, so do decision support systems for early detection of critical sailing situations, and, similarly, the sea state is needed for reliable calculations of fuel-performance during and after voyages. The gathering of sea state knowledge depends, however, on the stage of the marine operation. In the design stage, databases of ocean wave statistics are the prime source, while 'real-time stages' (e.g., actual operations and associated postvoyage evaluations) require the sea state to be estimated exactly when and where the operations take place. With a focus on real-time operations and post-evaluations, one means for the measuring of sea states is that of the wave buoy analogy, where the floating structure, often a ship-like vessel, is used as a wave buoy. Thus, the estimation principle relies on the combination of measurements of wave-induced motions of the vessel and a linear assumption, allowing the motion measurements to be modelled theoretically using transfer functions and a wave (energy density) spectrum.

Email address: udn@mek.dtu.dk (Ulrik D. Nielsen) 
This paper presents a study where wave-induced motion measurements from a larger in-service container ship are analysed and used with the wave buoy analogy for wave spectrum estimation. The study considers seven days of consecutive data obtained while the ship made an east-bound trip across the Pacific Ocean with measurements from the Sea of Japan to off Graham Island (Canada). In the present study, the wave buoy analogy is based on non-parametric (Bayesian) modelling, see Section 2, that facilitates an estimate of the full directional wave spectrum. The estimation is based on a simple instrumentation of the vessel, see Section 3, as a set of measurements from two accelerometers and one gyro forms the basis of the inverse problem together with strip theory calculations of the corresponding motion transfer functions. Specifically, the vessel's pitching motion and the horizontal and vertical accelerations at a position close to the ship's forward perpendicular have been measured.

\subsection{Scope and objective}

The calculation of the motion transfer functions requires exact knowledge of the vessel's advance speed, and the results of the wave buoy analogy may therefore be sensitive to variations in this parameter. For in-service data, this point is of importance, as it is interesting to consider exactly what speed to use; noting that a distinction between speed-over-ground (SOG) and speed-through-water (STW) should be made, since the two will generally be different because of sea current. In this discussion, it should be noted that advance speed basically enters the wave buoy analogy in two stages; (1) in the calculation of the transfer functions, as just reported, and, (2), when the Doppler Shift, which maps absolute frequency into encounter frequency, is introduced in the governing equations, as shown in Section 2. Moreover, the creditability of STW measurements by acoustic Doppler current profilers, also sometimes referred to by the Doppler Velocity Log, has been debated in recent years $[1,2,3,4,5]$. In a related context, the wave buoy analogy typically assumes stationary conditions during the recording period of the motion measurements. Stationary conditions, in the strict sense, is only the true reality in a very few cases, as smaller heading variations and changes in advance speed, not to mention changes in the sea state itself, will compromise the assumption.

It is a main task of the article to study the sensitivity to advance speed when wave spectra are estimated using the wave buoy analogy. In addition, the study discusses if the assumption about 'stationary conditions' seems reasonable during typical in-service conditions and, on a more general level, the article has as its overall objective to study the applicability of the wave buoy analogy for ships in service. It is noteworthy that it is an inherent concern that the ground truth of the sea state is never known during in-service conditions. In this study, additional knowledge about the sea state has been obtained from hindcast studies made by an external provider, and comparisons between the two sets of estimates - hindcast vs. wave buoy analogy - will be made. Other means and methods are available for on-site and real-time estimation of wave spectra $[6,7,8,9,10]$; however, this study has its focus exclusively directed towards the wave buoy analogy.

\subsection{A note about Bayesian modelling}

In the past, different estimation techniques have been studied in connection with the wave buoy analogy; an overview is given in [11]. In previous publications, comparisons have been made between the different estimation techniques and, based on results alone, [12] concludes that it is difficult to favour one technique from another when the Bayesian technique is compared to a parametric, optimisation-based method. On the other hand, from a theoretical point of view, the Bayesian technique offers more flexibility and few constraints and assumptions about the wave spectrum to be estimated. Good examples on the flexibility of the Bayesian method can be seen in [13, 14]. A new and (very) computationally efficient estimation technique has been presented by [15, 16] but due to its (strict) constraints, the Bayesian method is considered as a better choice in the analysis of in-service data, where fewer assumptions generally is the better. Altogether this is the reason behind the choice to focus the present study on the Bayesian technique.

\subsection{Composition of the article}

The remaining sections of the article are organised in the following way. The theoretical background of the study is given in Section 2 which includes a brief outline of the wave buoy analogy when it is applied together with Bayesian modelling, and, moreover, Section 2 includes the problem formulation in which the reasoning behind the sensitivity study on advance speed is given. Section 3 contains a description of the considered ship and data, including the origin of the data and how it has been processed. The results and corresponding discussions of the study are presented in Section 4, while a summary of the article and some concluding remarks are given in Section 5. 


\section{Theoretical background}

\subsection{Bayesian modelling}

The fundamental assumptions and the equations governing the wave buoy analogy have been reported in the literature previously, e.g., [17, 18, 19, 20, 11], and this section serves merely to indicate the most important aspects. The details can be found in $[19,20]$. It is noteworthy that, in the present subsection, sea current is not considered explicitly for what reason the distinction between SOG and STW is irrelevant.

The central assumption of the wave buoy analogy builds on linearity between waves and the wave-induced response of a vessel. In a frequency domain formulation, the combination with an assumption about stationarity implies the following model in which the (unknown) directional wave spectrum is $E\left(\omega_{e}, \mu\right)$,

$$
R_{i j}\left(\omega_{e}\right)=\int_{-\pi}^{\pi} H_{i}\left(\omega_{e}, \mu+\beta\right) \overline{H_{j}\left(\omega_{e}, \mu+\beta\right)} \cdot E\left(\omega_{e}, \mu\right) d \mu+\varepsilon_{i, j}
$$

Herein, $R_{i j}\left(\omega_{e}\right)$ is the response spectrum for responses $i$ and $j$, where $i$ and $j$ correspond to any set of measured ${ }_{44}$ responses; say, the horizontal acceleration and the vertical acceleration, respectively, in a specific point in the ship coordinate system. The corresponding theoretical response spectrum is obtained as the product between the directional wave spectrum $E\left(\omega_{e}, \mu\right)$ and the multiplication of the set of transfer functions $H_{i}\left(\omega_{e}, \beta+\mu\right)$ and $\overline{H_{j}\left(\omega_{e}, \beta+\mu\right)}$ for responses $i$ and $j$, with the bar denoting the complex conjugate. The mean wave-encounter angle is $\beta$ and the direction of waves relative to this angle is $\mu$, while the encounter frequency is $\omega_{e}$. The error between the measured spectrum and the theoretically calculated one is $\varepsilon_{i, j}$. It is noteworthy that Eq. (1) is usually formulated for three responses simultaneously which leads to a set of nine independent equations ${ }^{1}$. However, the directional wave spectrum is typically discretised into $K$ directions and, if the 360-degrees interval is spaced by, say, 10 deg., this results in $K=$ 36 unknown spectral components for any given frequency. Consequently, Eq. (1) expresses a highly underdetermined equation system that cannot be solved by minimising the error $\varepsilon$, as the corresponding least squares problem is ill-posed. Instead, Bayesian modelling can be applied to solve the equation system. The main points of Bayesian modelling are presented below but, before this, the effect of advance speed deserves special attention.

The equation system in Eq. (1) is formulated in the 'encounter domain' as the spectral densities of the wave spectrum depend on the encounter frequency $\omega_{e}$, which itself is dependent on the vessel's advance speed and the wave encounter angle. As a consequence, the absolute frequency must be used instead, and it is therefore necessary to introduce the Doppler Shift. Thus, the mapping of the absolute frequency, sometimes also referred to by the intrinsic frequency, $\omega$ (of a progressive wave) to the encountered frequency $\omega_{e}$ is given by,

$$
\omega_{e}=\omega-\omega^{2} \psi, \psi=\frac{U}{g} \cos \mu
$$

if the "observer", i.e. the ship, moves with advance speed $U$ and at an angle $\mu$ relative to the progressive wave; $g$ is the acceleration of gravity. It is noteworthy that deep-water conditions are assumed in this study; thus relating wave number $k$ to frequency by $k=\omega^{2} / g$. In practice, the introduction of the Doppler Shift is not without complications in problems of ship motion dynamics [21, 22, 23, 24, 25], but this is beyond the scope of the present paper.

Four points are noteworthy in connection with Eq. (1), when Bayesian modelling is used to solve the equation system: (i) The error $\varepsilon_{i, j}$ is modelled as Gaussian white noise with zero mean and (pre-conditioned) standard deviation [19]. (ii) The Doppler Shift, Eq. (2), is directly incorporated into the governing equation system, Eq. (1), to introduce advance speed. This means that the directional wave spectrum is solved for the absolute wave frequency $\omega$ rather than the encounter frequency $\omega_{e}$. (iii) A non-negativity constraint is imposed on the discrete components of the directional wave spectrum. (iv) Additional equations can be established by assuming the directional wave spectrum to be a smooth (and continuous) function for variations with frequency and direction. The compromise between agreement of the data with the solution on the one side versus smoothing on the other side is controlled by the so-called hyperparameters. The values of the hyperparameters are found by optimising a certain criterion [26]. In principle, the optimisation must

${ }^{1}$ Cross spectral analysis on three discrete-time motion signals leads to 3 real-valued spectra and $6(=3 \times 2)$ complex-valued spectra with both real and imaginary parts. 
be made every time a specific wave spectrum is estimated on the basis of a set of motion measurements, recorded over, say, 30 minutes. However, in this study it is decided to "pre-calibrate" the hyperparameters $[27,28]$ as this greatly increases the computational efficiency.

\subsection{Problem formulation - Inclusion of sea current}

A ship in service often navigates ocean waters where the rate of sea current is not negligible compared to the ship's advance speed. Consequently, two speed measures exist; the vessel's speed-through-water and its speed-over-ground. The existence of sea current in connection with applications of the wave buoy analogy has not been given much attention in the past, and this is partly the motivation of the present study. The following considerations are based on the situation of a uniform current of rate $V$ which is superimposed on a (short-crested) wave system in deep-water. Leaving the ship out, for wave systems in ocean waters with a superimposed current, the apparent frequency $\omega_{a}$, as seen by an observer from a fixed point in space, differs from the intrinsic frequency $\omega$ by the product of the wave number vector $\mathbf{k}$ and the velocity vector $\mathbf{V}$ of the current, i.e. $\omega_{a}=\omega+\mathbf{k V}[29,30,31]$. The presence of a current does not change the apparent frequency, but changes the wave height and thus the wave energy spectral density. The current-induced change of the wave spectral density is beyond the scope of this study, and the point to note is that if the observer instead moves in space, it is the relative velocity between the current and the observer that must be used for the frequency mapping. Hence, for ships sailing in seaways with a superimposed current, the Doppler Shift, Eq. (2), must be calculated using the vessel's speed-through-water (STW) as the advance speed $U$. Similarly, when it comes to the calculation of the motion transfer functions, the same reasoning dictates that the calculations must be made using the vessel's STW as the advance speed. Altogether, it can be concluded that, in applications of the wave buoy analogy for ships in service, the vessel's STW is the important speed measure.

As was indicated in the introduction, STW is typically measured using acoustic Doppler current profilers (ADCP). The working principle of this type of sensor is not considered here, but it is important to address the fact that several studies are concerned with the accuracy of the sensor [1,2,3,4,5]. Consequently, it becomes of interest to study the influence of (in)accurate STW measurements when the wave buoy analogy is applied with data from an in-service ship having its speed-through-water measured by an ADCP.

\section{Case ship and in-service data}

The case ship is a 7,200 TEU container vessel. The vessel's main particulars are listed in Table 1.

Table 1: Main particulars of the example ship

\begin{tabular}{lr}
\hline Length between perpendiculars, $L_{p p}$ & $332 \mathrm{~m}$ \\
Breadth moulded, $B_{m}$ & $42.8 \mathrm{~m}$ \\
Design draught, $T_{d}$ & $12.2 \mathrm{~m}$ \\
Deadweight (at $T_{d}$ ), & 76,660 tonnes \\
Block coefficient, $C_{B}$ & 0.65 \\
\hline
\end{tabular}

As part of a measurement campaign, the ship was installed with a motion sensor (XSENS, MTi-30-6A5G4), see Figure 1 (a). The sensor was mounted in a bosun store close to the bow, off the centreline, with the exact position known by the authors. The particular sensor provides drift-free 3D orientation as well as calibrated 3D acceleration, 3D rate of turn (rate gyro) and 3D earth-magnetic field data. For sea state estimation, it was decided to focus exclusively on using three of the motion components; the horizontal and vertical accelerations, respectively, and the pitch motion. The corresponding transfer functions have been calculated with an in-house linear strip theory code [32]. The measurements of this study were recorded on an east-bound route across the Pacific Ocean, see Figure 1 (b).

The sensor readings of the speed-logs (STW as well as SOG) can be seen in Figure 2, where the x-axis represents the date and time stamps in the format 'month-day UTC-time' in the year 2016. According to the logs, the advance speed was kept nearly constant around 21 knots for the whole period, and the vessel's draught readings were (almost) constant too with a mean draught of $14.1 \mathrm{~m}$. Although being 'nearly constant' it is interesting to see that the SOG exhibits more variations around its mean than the STW, likely as a result of increased sea current rate encountered 

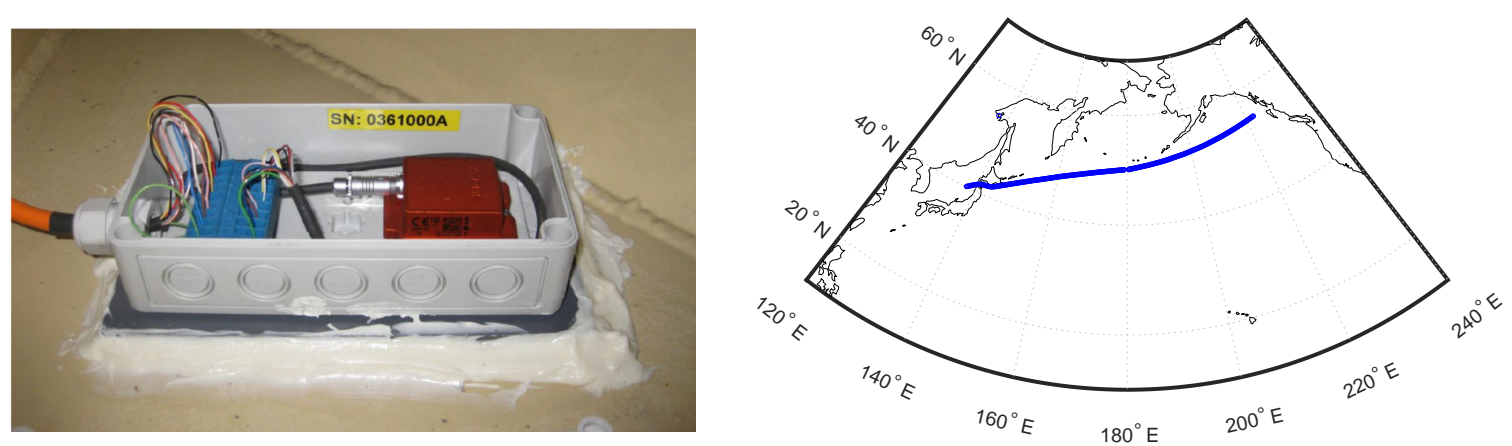

Figure 1: (a) The motion sensor inside sensor box. (b) The analysed measurements have been recorded during an east-bound voyage across the Northern Pacific.

from time to time. This observation and its consequence will be further discussed in Section 4. The loading condition, here represented by the mean draught, is also important, and a ship's wave-induced motion dynamics will be affected by variation and uncertainties associated with it. However, in this study, the sensitivity to loading condition is not studied.

During the voyage, a total of $336(=7 \times 48)$ 30-minutes motion samples were collected. After the initial ("raw") sampling at $100 \mathrm{~Hz}$, the motion recordings were resampled to $5 \mathrm{~Hz}$ for the pure sake of computational efficiency. Next, for each set of the 30-minutes motion samples, the cross power spectral density of the pairs of motion components was calculated using Welch's averaged, modified periodogram method. The resulting set of nine (cross) spectra, as used for wave spectrum estimation for a single motion sample, was limited to the encounter-frequency interval $[0.01 ; 0.30] \mathrm{Hz}$, spaced $0.005 \mathrm{~Hz}$, emphasising that no significant (wave-induced) motion occurs outside this interval. Finally, for each 30-minutes motion sample, the directional wave spectrum has been estimated, cf. Section 2, using a discretisation with $M=30$ absolute wave frequencies and $K=36$ (relative) wave directions on the intervals $\omega=$ $[0.01,0.30] \mathrm{Hz}$ and $\mu=]-180 ;+180] \mathrm{deg}$, respectively; noting that, for the relative wave direction, equivalently wave encounter angle, $\mu=180 \mathrm{deg}$ is head sea, $\mu=0 \mathrm{deg}$ is following sea, while ' + ' and '-' are used to indicate if waves approach on the starboard or port side, respectively. Two arbitrarily selected examples of estimated directional wave spectra are shown in Figure 3.

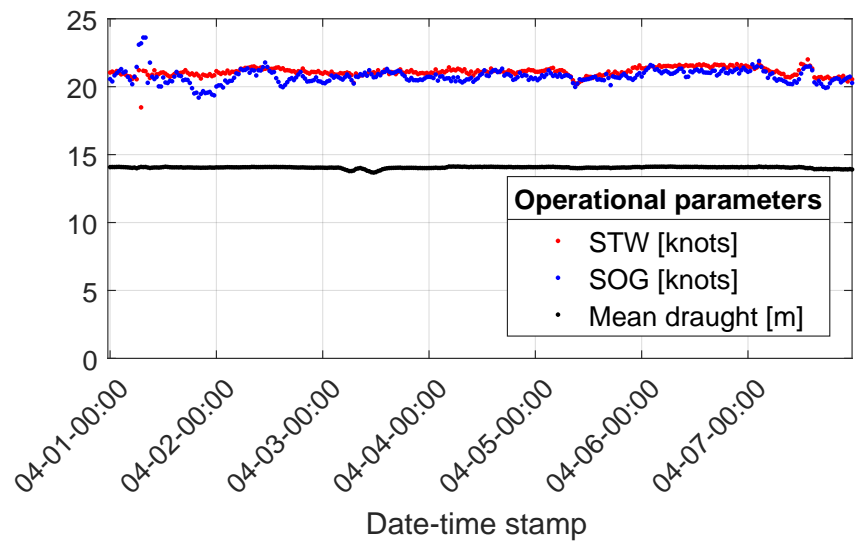

Figure 2: Measurements of advance speed during the voyage, using an acoustic Doppler current profiler (ADCP) for speed-through-water (STW), while GPS provides speed-over-ground (SOG). 
Dir. wave spec. (04-04-08:00)

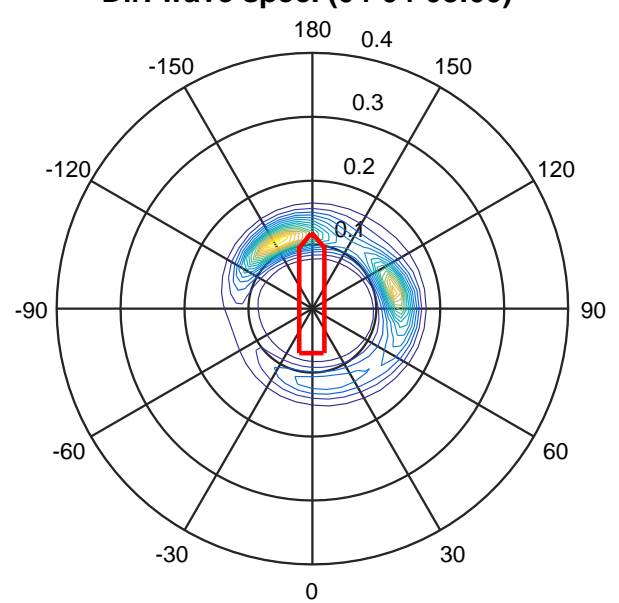

Dir. wave spec. (04-07-09:00)

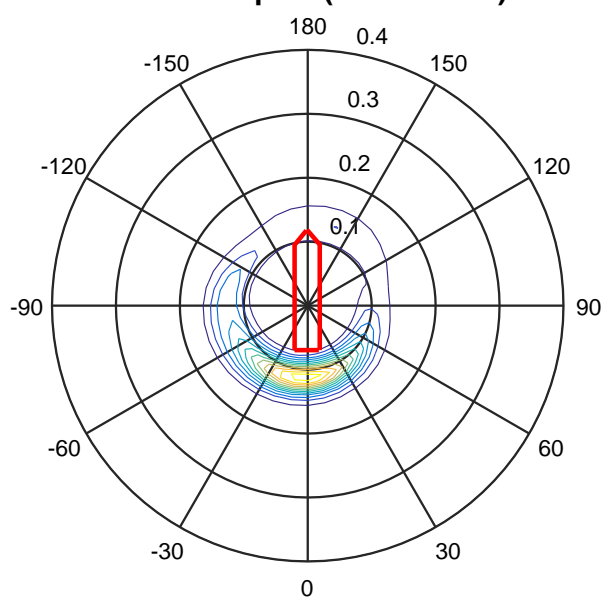

Figure 3: Examples of directional wave spectra. In the polar plots, the waves are shown as "approaching from" and their relative direction to the ship is visualised.

\section{Results and discussions}

In Section 2 it was pointed out that the results of the wave buoy analogy depend on speed-through-water (STW), since the motion transfer functions of the vessel as well the Doppler Shift must be calculated according to STW. For a ship in service, the value of STW comes from the ADCP and, in the following, two sets of estimates will basically be presented. Initially, in Subsection 4.2, the value of STW will be based on the actual measurement from the ADCP while later, in Subsection 4.3, the value of STW will be inconsistent with the measurements from the ADCP. Before the results are presented, the next subsection includes the definitions of the wave parameters which will later be used as metrics to compare the wave spectrum estimates by the wave buoy analogy and somewhat corresponding estimates from a hindcast study.

\subsection{Integral wave parameters and hindcast wave data}

In essence, the outcome of the wave buoy analogy, based on Bayesian modelling, is the directional wave spectrum. The spectrum can be associated with a set of integral wave parameters; for instance, the significant wave height $H_{s}$, the mean wave period $T_{s}$, the mean energy period $T_{E}$, the peak period $T_{p}$, the relative mean wave direction $D_{s}$, and the relative direction of the spectrum peak $D_{p}$. The definitions of the parameters are:

$$
\begin{aligned}
H_{s} & =4 \sqrt{m_{0}} \\
T_{s} & =2 \pi \frac{m_{0}}{m_{1}} \\
T_{E} & =2 \pi \frac{m_{-1}}{m_{0}} \\
T_{p} & =\frac{2 \pi}{\omega_{p}}, \quad F\left(\omega_{p}\right) \equiv \max _{\omega} F(\omega) \\
D_{s} & =\arctan (d / c) \\
D_{p} & =\mu_{p}, \quad E\left(\omega, \mu_{p}\right) \equiv \max _{\mu} E(\omega, \mu)
\end{aligned}
$$


where

$$
\begin{aligned}
m_{n} & =\int_{0}^{\infty} \omega^{n} F(\omega) d \omega \quad n=\{-1,0,1,2\} \\
F(\omega) & =\int_{-\pi}^{\pi} E(\omega, \mu) d \mu \\
d & =\int_{-\pi}^{\pi} \int_{0}^{\infty} E(\omega, \mu) \sin \mu d \omega d \mu \\
c & =\int_{-\pi}^{\pi} \int_{0}^{\infty} E(\omega, \mu) \cos \mu d \omega d \mu
\end{aligned}
$$

As indicated previously, hindcast (HC) wave data has been provided by an external provider in connection with general tasks related to vessel and fleet performance analysis by the company of the second author. The data can be used for comparison with the results by the wave buoy analogy (WBA), but it must be noted that both sets of results, WBA as well as HC, are merely estimates of the sea state, since the ground truth is unknown.

The HC wave data is composed by one set of wave parameters for swells and one set for wind sea, respectively, while the directional wave spectrum is not available. The available parameters for each of the components are significant wave height $H_{s}$, mean energy period $T_{E}$ and mean (relative) direction $D_{s}$. For comparative reasons, it is beneficial to calculate equivalent wave parameters of the total wave system being the combination of the wind sea and the swell components. In this case, the (total) significant wave height is obtained by

$$
H_{s, H C}=\sqrt{H_{s, \text { wind }}^{2}+H_{s, \text { swell }}^{2}}
$$

The "total mean" relative direction $D_{m, H C}$ is approximated by introducing a weighted average considering the relative direction of the individual components (swell and wind sea) together with their energy content represented by the significant wave height. In this calculation, special care must be shown because directionality is circular - that is, defined on the interval ${ }^{2}[0,360$ [ deg where $0 \mathrm{deg}$ and $360 \mathrm{deg}$ are the same point - and so this must be accounted for in the calculation. The weighting is according to ratios of the squared values of significant wave height and, schematically, the definition of $D_{m, H C}$ is,

$$
D_{m, H C}=\frac{H_{s, \text { wind }}^{2}}{H_{s, \text { tot }}^{2}} \cdot D_{s, \text { wind }}+\frac{H_{s, \text { swell }}^{2}}{H_{s, \text { tot }}^{2}} \cdot D_{s, \text { swell }}
$$

It is possible to approximate the "total mean" period $T_{m, H C}$ in a similar way, and the calculation follows from

$$
T_{m, H C}=\frac{H_{s, \text { wind }}^{2}}{H_{s, \text { tot }}^{2}} \cdot T_{E, \text { wind }}+\frac{H_{s, \text { swell }}^{2}}{H_{s, t o t}^{2}} \cdot T_{E, \text { swell }}
$$

It is noteworthy that, intentionally, the index on the total mean relative direction and on the total mean period for the HC results is ' $m$ ' and not 'E', since the derived results $D_{m, H C}$ and $T_{m, H C}$, respectively, in Eqs. (14) and (15) are not equivalent with the (spectral) mean relative direction $D_{s}$, Eq. (7), and the (spectral) mean energy period $T_{E}$, Eq. (5). In the following, comparisons between the WBA results and the HC results will be made on the basis of the derived parameters, although the parameters are not equivalent from a theoretical point of view.

\subsection{ADCP-consistent speed-through-water}

In the initial part of the study, the STW, as used with the wave buoy analogy, is set in accordance with the ADCP measurements, cf. Figure 2. Specifically, a constant value of 21.0 knots is used, although smaller fluctuations around this value can be seen from the sensor readings.

The estimates of significant wave height can be seen in Figure 4, emphasising that the total significant wave height (wind sea + swell) is considered. It is noted that a small "gap" exists in the HC results during the hours 18:30-23:30 on 04-04; the explanation for the missing values are, however, unclear. Generally, the agreement between the results are reasonable, and the trends in the development of the (total) significant wave height are quite similar. 


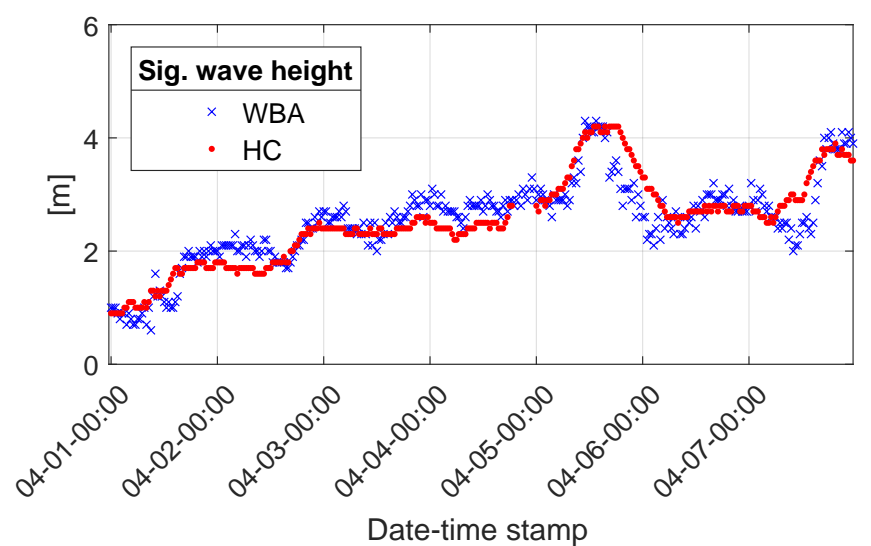

Figure 4: Estimates of significant wave height, derived from Eq. (3), for the wave buoy analogy (WBA) and corresponding estimates obtained by hindcast (HC), cf. Eq. (13).

The significant wave height represents a measure of the total energy (per square meter of sea surface) of a wave system. The distribution of energy (density), on the other hand, can be represented by wave periods. Figure 5 shows the peak period $T_{p}$ and the mean energy period $T_{E}$ for the WBA results together with the approximated mean energy period of the total wave system for the $\mathrm{HC}$ results. In accordance with the last paragraph of subsection 4.1, the comparison of $\mathrm{HC}$ and WBA is made for $T_{E, W B A}$ and $T_{m, H C}$ although, theoretically speaking, the two are not fully equivalent. It is noted that the agreement between results of the WBA and by HC is reasonable for some portions of the data, while, for other parts, the picture is more blurred. Generally, the tendency is that $T_{E, W B A}$ is larger than $T_{m, H C}$. Moreover, it is seen that $T_{E, W B A}$ is larger than $T_{p, W B A}$, which is inconsistent with findings for standard parameterised unimodal wave spectrum shapes (e.g. JONSWAP or Pierson-Moskowitz). Both of these observations is likely a result of the low-pass filtering characteristic of a ship, as discussed in earlier publications about the use of ships as wave buoys, e.g. [33, 34]. Consequently, the (frequency) wave spectrum of the WBA, cf. Eq. (10), may sometimes exhibit too little "skewness" in its spectrum shape, which is illustrated in Figure 6 for a selected case from 04-04-12:00. In the specific case, it is evident that the peak frequency is larger than the mean (energy) frequency, since the high-frequency tail, typically observed for ocean wave spectra, has ("erroneously") been cut off. Although the low-pass filtering

${ }^{2}$ In practice, the interval is ]-180,180] deg, see Section 3, but the overall problem is the same.

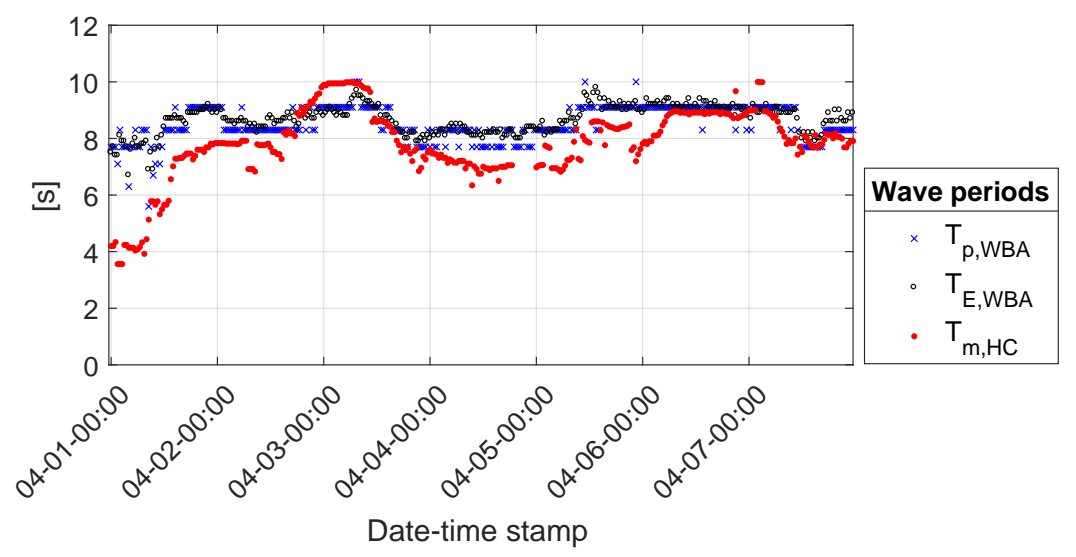

Figure 5: Estimates of the peak and the mean energy period for the wave buoy analogy (WBA) using Eqs. (6) and (5), respectively. For the hindcast (HC) results, the derived "total" mean energy period $T_{m, H C}$, Eq. (15), is shown. 


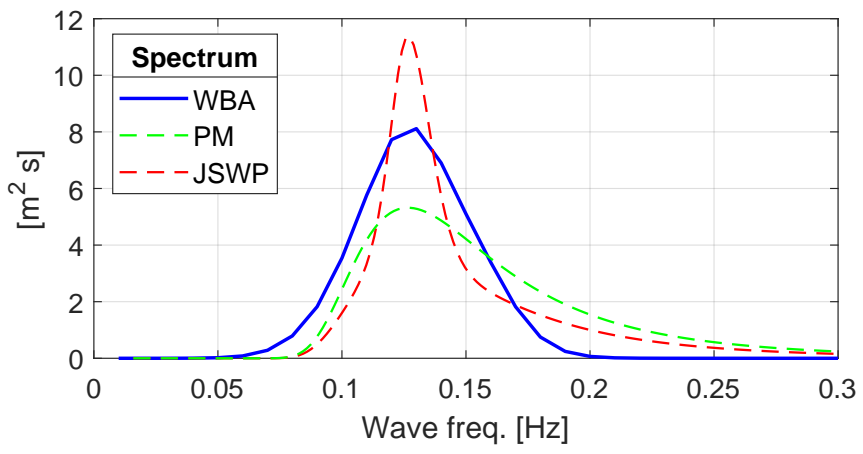

Figure 6: Frequency wave spectrum (Eq. 10) as estimated by the WBA on 04-04-12:00. Basically, any ship is a low-pass wave filter and, like in this case, the high-frequency tail may be "cut off" since the particular vessel does not respond to waves with a frequency higher than about 0.20 Hz. For comparison, standard spectra in terms of a Pierson-Moskowitz (PM) and a JONSWAP (JSWP) with the same integral wave parameters are included.

characteristics of a ship is an inherent drawback of the wave buoy analogy, it must be emphasised that the result of the WBA with respect to wave periods, cf. Figure 5, is generally within the accuracy ( $\sim 1$ second) of the HC result itself, without saying what the ground truth is.

Estimates of the (relative) wave direction can be seen from Figure 7, where the different parameters can be compared. Taking note of the inconsistency in the parameters of the WBA and those of the HC results, the most indicative comparison to make is among the mean relative direction $D_{s, W B A}$ and $D_{m, H C}$. In this case, a reasonable agreement is found between the results by the WBA and the HC for many portions of the data; especially by keeping in mind that directionality is circular; that is, $-180 \mathrm{deg}$ and $180 \mathrm{deg}$ are equivalent.

As the final comparison, Figure 8 shows a set of comparative plots, where ratios of the parameters from the WBA and $\mathrm{HC}$ are included for the significant wave height, the wave period, and the relative wave direction. Specifically, the ratios are formed as

- $\frac{H_{s, W B A}}{H_{S, H C}}$ for the significant wave heights using Eqs. (3) and (13) for the WBA and the HC results, respectively

- $\frac{T_{E, W B A}}{T_{m, H C}}$ for the wave periods using Eqs. (5) and (15) for the WBA and the HC results, respectively

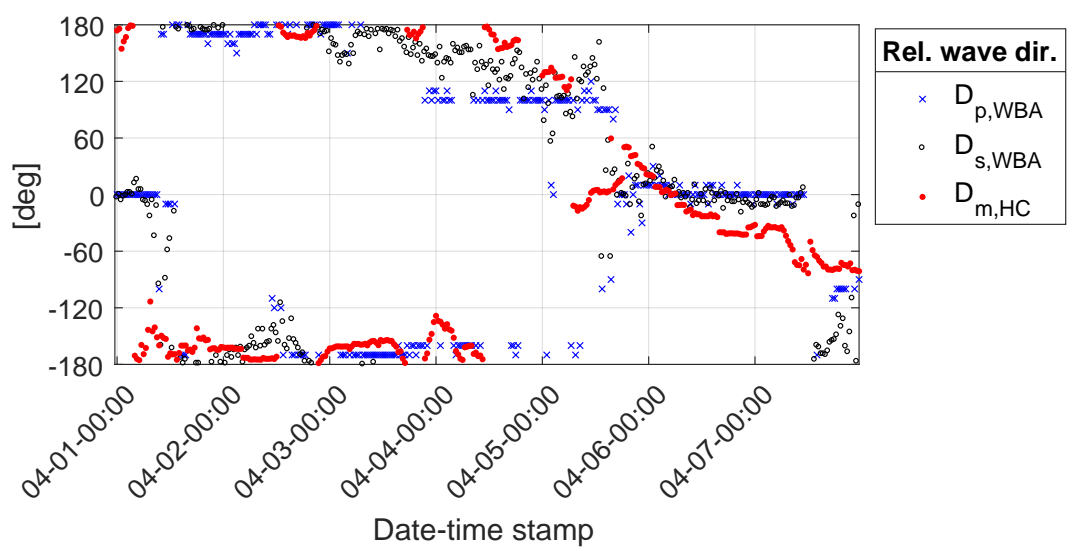

Figure 7: Estimates of the relative wave direction(s) for the wave buoy analogy (WBA) using Eqs. (7) and (8). For the hindcast (HC) results, the derived "total" mean relative direction $D_{m, H C}$, cf. Eq. (14), is shown. 

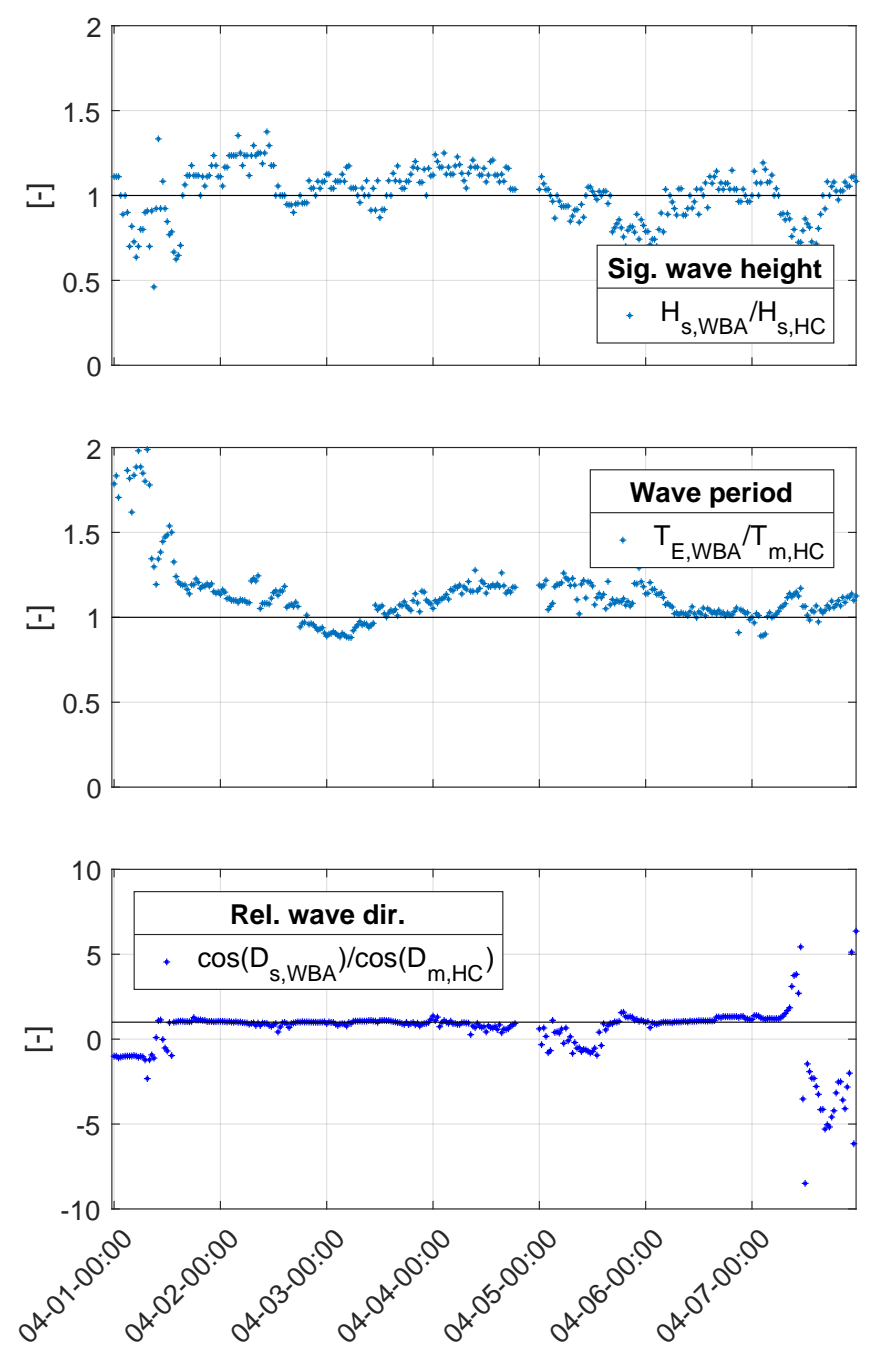

Figure 8: Non-dimensional ratios between wave parameters obtained from the wave buoy analogy (WBA) and from hindcast (HC); a perfect agreement implies unity (1.0).

- $\frac{\cos \left(D_{s, W B A}\right)}{\cos \left(D_{m, H C}\right)}$ for the relative wave directions using Eqs. (7) and (14) for the WBA and the HC results, respectively

Thus, the individual ratio is unity in case of identical values by the WBA and the HC, and, moreover, it is noted that the circular behaviour of directionality is considered by applying cosine on the directions. From the plots in Figure 8 it can be seen that the agreement between the two sets of estimates (WBA vs. HC) is, in fact, acceptable. Although neither of the sets should be taken as the ground truth, the averages of the relative deviations, formed by $\delta_{\{H s, T, D\}} \equiv\left(\frac{W B A-H C}{H C}\right)$, are $\delta_{H_{s}}=0.01(0.15), \delta_{T}=0.14(0.21)$, and $\delta_{H_{s}}=-0.50(1.5)$ for the significant wave height, the wave period, and the relative wave direction, respectively. The number in the parenthesis is the corresponding standard deviation. The values of the relative deviations confirm the reasonable agreement; noting that the relatively large deviation on the relative wave direction is a result of "outliers" in the beginning and at the end of the data stream.

\subsection{Sensitivity to $S T W$}

In this subsection, the sensitivity of the WBA results to the vessel's speed-through-water (STW) is studied. In the former subsection, it was concluded that the agreement between the results of the wave buoy analogy and (somewhat) 

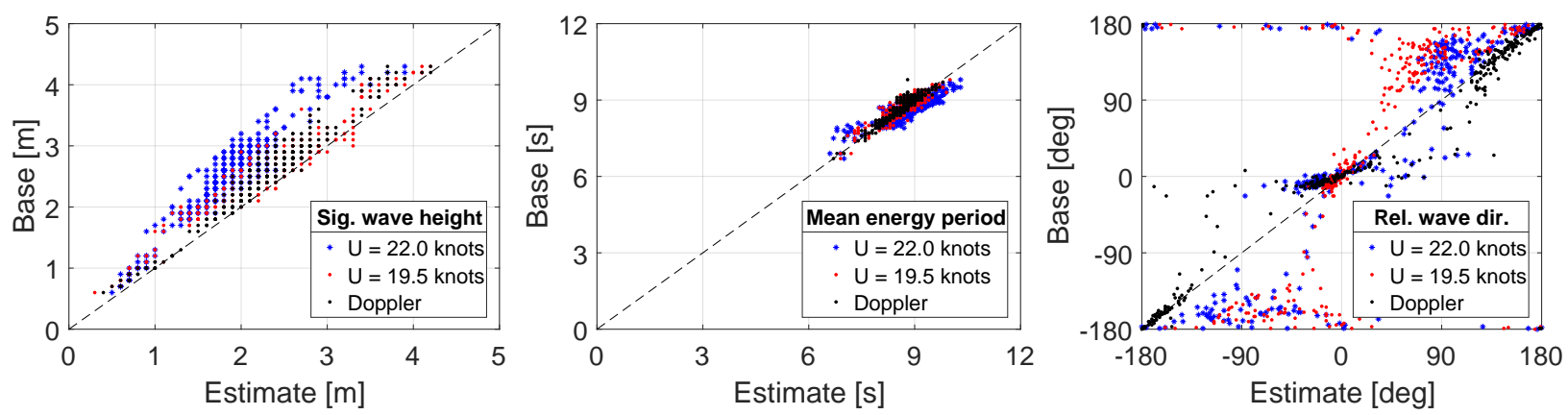

Figure 9: Comparisons for significant wave height $H_{s}$ (left-side plot), mean energy period $T_{E}$ (middle plot), and mean relative wave direction $D_{s}$ (right-side plot). Note, 'Base' is the result using the actually measured STW ( $U=21.0$ knots), while 'Estimate' is obtained using the STW reported in the legend.

corresponding results of a hindcast study was acceptable. In the present subsection, the hindcast results are not used; instead, the results of the wave buoy analogy (WBA) from the previous subsection are used as the comparative basis.

In the preceding subsection, the vessel's advance speed, as used with the wave buoy analogy, was consistent with the STW measurements by the ADCP. In this part of the study, two cases with inconsistent STWs are considered. Thus, the STW is set to 19.5 knots in the first subcase, and 22.0 knots in the second subcase; meaning that incorrect velocities are in fact used for the calculation of the transfer functions and the Doppler Shift.

The results corresponding to the two subcases are shown in Figure 9, noting that the figure presents comparisons for the significant wave height $H_{s}$ as the left-side plot, while comparisons for the mean energy period $T_{E}$ and for the mean relative direction $D_{s}$ are seen as the middle plot and the right-side plot, respectively. Basically, the individual plots show the correlation between the base measure and the "new" estimates, where the base values, being it $H_{s}, T_{E}$ or $D_{s}$, are identical to the results from the previous subsection, with ADCP-consistent STW (i.e., $U=21.0$ knots). The new estimates, on the other hand, correspond to the two sets of ADCP-inconsistent STWs having legends ' $\mathrm{U}=$ 22.0 knots' and ' $U=19.5$ knots'. It is noted that a third subcase is included in the plot with legend 'Doppler'. The meaning of this subcase is explained further below.

The plots in Figure 9 show that variations in the advance speed (i.e. STW), relative to the "true" value, affect the results of the wave buoy analogy quite much. In the particular case, it can be seen that the largest effect is observed for a speed increase (' $\mathrm{U}=22.0$ knots') for both the significant wave height and the relative mean wave direction, while the mean energy period is generally less affected, irrespectively of the speed variation. It is interesting to see that the significant wave height (almost) consistently is estimated with lower values compared to the base. This observation, in combination with the fact that the mean energy period is more consistent among the speed cases, indicates that energy (density) is generally missing in the tail regions of the estimated wave spectra.

It is clear from the results in Figure 9 that outcomes of the wave buoy analogy will be (negatively) affected if the STW is not accurately measured by the ADCP. The sensitivity to STW of the wave buoy analogy will generally be governed by the transfer functions and the Doppler Shift, cf. Eq. (2). For the present results, the sensitivity is dominated by the use of a set of transfer functions which is calculated at the wrong advance speed. This can be seen from the third subcase, with legend 'Doppler', in Figure 9. This subcase shows the result of the wave buoy analogy when the transfer functions have been calculated with the correct STW $(U=21.0$ knots $)$, whereas the Doppler Shift, Eq. (2), is based on an incorrect STW with $U=22.0$ knots. Thus, it can be observed that the estimates of this third subcase are fairly consistent with the "original" case, cf. subsection 4.2.

\subsection{Influence of sample length}

All of the preceding results were produced using 30-minutes motion samples for which stationary conditions were assumed. During in-service conditions, two related but "counteracting" concerns arise with respect to the length of the motion sample, keeping in mind that the wave buoy analogy relies on a frequency domain analysis of a set of time series. First, the specific motion samples must be sufficiently long to contain enough frequency components to ensure reliable spectral calculations. Second, the longer the samples, the less good/uncertain is the assumption 


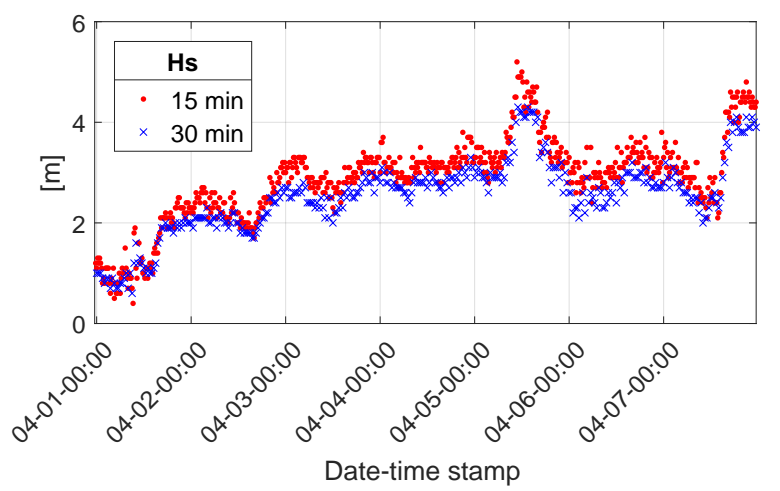

Figure 10: Estimates of significant wave height when different sample lengths are used.
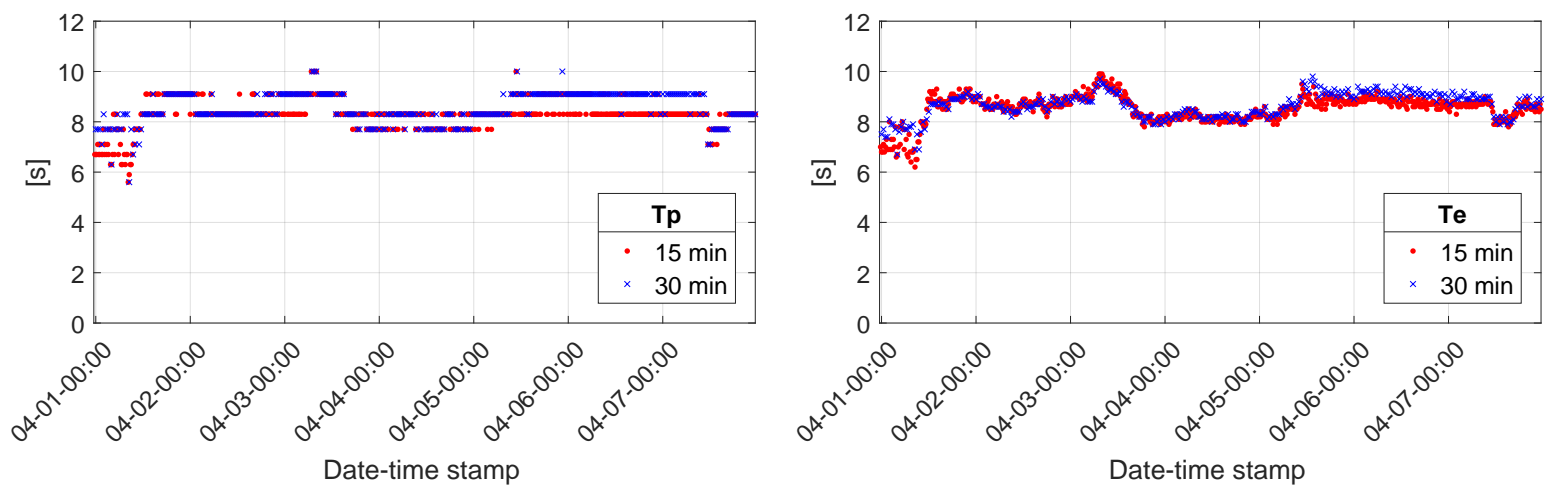

Figure 11: Estimates of the peak period (left-side plot) and the mean energy period (right-side plot) when different sample lengths are used.

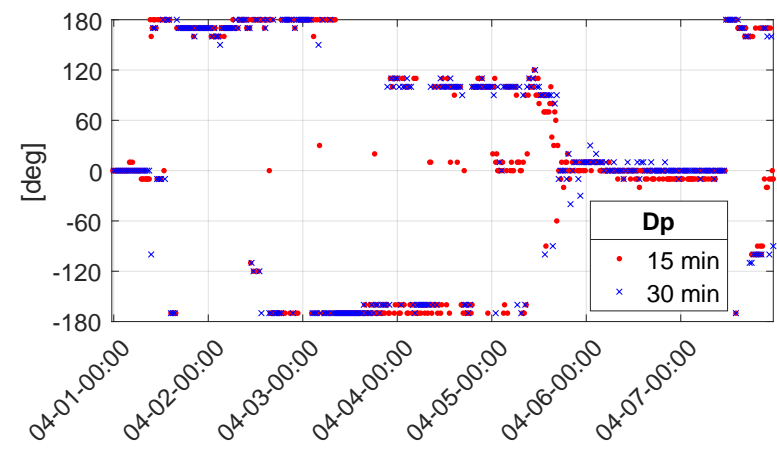

Date-time stamp

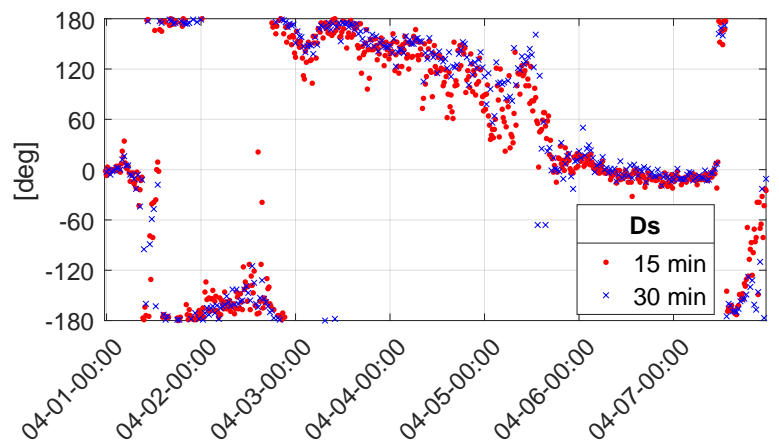

Date-time stamp

Figure 12: Estimates of the relative direction of the spectrum peak (left-side plot) and mean relative wave direction (right-side) when different sample lengths are used.

about stationary conditions. In this study, the samples were of length 30 minutes, as given by the default setting of the sensor installation. It seems reasonable to assume that a 30-minutes sample length is likely the maximum within which stationary conditions can be assumed; especially for a vessel that steams ahead with 21 knots. On the other hand, it would be interesting to see the effect (if any...) if the sample length was reduced to, say, 15 minutes. In this case, the main results from the wave buoy analogy are presented in Figures 10-12. In the plots, results of the wave parameters 
are compared when sample lengths of 15 minutes and 30 minutes, respectively, are used. Practically, each 30-minutes sample was divided into two 15-minutes samples comprising the first and second half of the 30-minutes sample. In each of the plots (Figs. 10-12), there are therefore twice as many points for the 15-minutes samples compared to the number of markers for the 30-minutes samples. Note, the STW was $U=21.0$ knots in all calculations which means that the results of the 30-minutes cases correspond to those in Subsection 4.2. The general observations from the plots are the following:

- Significant wave height $H_{s}$ (Fig. 10). The results from the 15-minutes samples are almost consistently 10-15\% larger, and more in some cases. The relatively poor agreement seems not to be correlated with the (dis)agreement of any of the other parameters, neither period nor direction. The somewhat peculiar observation is likely a result of non-stationary conditions, but the precise explanation cannot be given.

- Wave periods $T_{p}$ and $T_{E}$ (Fig. 11). The results, $15 \mathrm{~min}$. vs. $30 \mathrm{~min}$., are fairly identical, and there appears to be no trend in the observed (smaller) differences; neither for $T_{p}$ nor for $T_{E}$.

- Relative wave directions $D_{p}$ and $D_{s}$ (Fig. 12). The consistency between the two sample lengths, 15 min. vs. 30 min., is good for most portions of the data. However, it appears that there is a tendency for some of the 15-minutes cases to show a mean relative direction $\left(D_{s}\right)$ that indicates waves being "less head sea".

It is difficult to draw specific conclusions from Figures 10-12, but the findings indicate that conditions are not truly stationary during the measurements. However, while the observations may be a result of a lack of stationarity, it is also well-known that short-term (aleatory) variability in sea states themselves affect the outcome of the wave buoy analogy $[35,36]$. Therefore, the central point from this sub-study is that more detailed analyses are needed to investigate and possibly clarify at what "level of non-stationarity" the results of the wave buoy analogy will become (significantly) compromised. Such analyses are, however, beyond the scope of the present work and, as a supplementary comment, another point of the sub-study is that the significance of the lack of stationary conditions, although of importance from a theoretical point of view, is likely small in relation to actual and practical use of the wave buoy analogy if/when the results are as presented in this particular study.

\section{Summary and concluding remarks}

In the article, wave-induced motion data from a larger, in-service container ship was applied for wave spectrum estimation. Thus, the ship has itself been considered as a (sailing) wave buoy. In this study, the wave buoy analogy was formulated using Bayesian modelling while the wave-induced motion components were pitch together with the horizontal and vertical accelerations in a position close to the forward perpendicular. The investigated data was collected during a seven-days crossing of the Pacific Ocean.

The wave buoy analogy is dependent on accurate calculations of the motion transfer functions, which should apply to the exact operational condition. Consequently, the vessel's speed-through-water (STW) is an important parameter, noting that generally, STW and SOG (speed-over-ground) are different because of the possible existence of a sea current. In the wave buoy analogy, STW plays another important role as it is in use when encounter frequencies are mapped to absolute frequencies, or vice versa, through the Doppler Shift.

In a comparative study, the article shows that estimates of sea state parameters (wave height, period and relative wave direction) by the wave buoy analogy are fairly consistent with estimates obtained from a hindcast study made by an external provider; noting that neither hindcast nor the wave buoy analogy represent the ground truth. For most portions of the data, the relative deviations in sea state parameters were up to about $10 \%$, when the advance speed of the vessel was set according to the acoustic Doppler current profiler (ADCP) of the vessel. During a sensitivity study on advance speed, it was shown that accurate measurements of STW are fundamental, as erroneous measurements of the speed may have a severe effect on the results of the wave buoy analogy. This finding is of interest since measurements of STW, by the ADCP, may not always be reliable as discussed in other literature. The problem is, however, that the ground truth is rarely known during in-service conditions, and it could therefore be of relevance to "integrate" the wave buoy analogy with approaches for improved speed-trough-water readings, e.g. [1, 4].

The article also discussed the assumption about stationary conditions during the sample period, albeit it was difficult to conclude anything from a smaller sub-study made in this connection. Generally, it will be much dependent 
on the actual data, whether or not stationary conditions is a reasonable assumption. It seems, like found in many other studies related to ship motion dynamics in real seaways, that sample periods between 15 and 30 minutes may contain sufficient wave components and still be of such a (short) duration that the sea state remains constant. In this context, there is no need to say that the operational parameters, notably advance speed and vessel heading, must remain constant as well.

Finally, as the consolidating remark, this study has shown that the wave buoy analogy can be applied together with ships in service for the provision of directional wave spectra at the exact point of operation.

\subsection{Future studies}

It is expected that additional data from the particular vessel will become available. This should lead to similar studies as presented in the article. In line with this, it will be important to investigate for the effect of severe waveinduced motions, as the wave buoy analogy, in theory, can be used only during mild wave conditions where the linear assumption holds; noticing that in the present study wave heights in excess of 4 metres were estimated.

Wave-current interactions and their effects have been studied in several works [29, 30, 31, 37]. This study was restricted to merely consider the effect of sea current on the Doppler Shift and the motion transfer functions, while the current-induced changes of the wave spectral densities were neglected. In future work on the wave buoy analogy, it is suggested to include the modifications to the wave spectrum as a result of sea current, and, moreover, it could be relevant to study the effect of intermediate water depths.

As a final note, the present study confirms that simple (low-cost) sensor instrumentation on ships facilitates reliable wave spectrum estimation. In his role as an industrial representative, the second author of this paper sees the derived principles as a practical solution to collect additional wave data valid at the exact point of operation. This goes hand in hand with other studies related to e.g. more trustworthy prediction of speed-through-water allowing an improved understanding of vessel performance.

\section{Acknowledgment}

The authors would like to acknowledge that vessel motion data was collected together with Propeller Control ApS through the Blue Innoship Project 2, which was supported by the Danish Innovations Fund, the industrial partners and the Danish Maritime Fund. Thanks are directed to Jos Koning (MARIN) for answers and clarifications about the sensor installations and data collection, and Jørgen Juncher Jensen (DTU) is acknowledged for general discussions. The work by the first author has been supported by the Research Council of Norway through the Centres of Excellence funding scheme, Project number 223254-AMOS.

\section{References}

[1] M. Antola, A. Solonen, J. Pyorre, Notorious Speed Through Water, in: Proc. of 2nd Hull Performance and Insight Conference, Ulrichshusen, Germany, 2017.

[2] T. Hasselaar, J. den Hollander, Uncertainty of Ship Speed Determination when Sailing in Waves, in: Proc. of 2nd Hull Performance and Insight Conference, Ulrichshusen, Germany, 2017.

[3] J. Taudien, S. Bilén, Quantifying Long-Term Accuracy of Sonar Doppler Velocity Logs, IEEE Journal of Oceanic Engineering 43 (2018) $764-776$.

[4] A. Oikonomakis, R. Galeazzi, J. Dietz, U. Nielsen, K. Holst, Application of Sensor Fusion to Drive Vessel Performance, in: Proc. of 4th Hull Performance and Insight Conference, Gubbio, Italy, 2019.

[5] G. Prytz, R. Gangeskar, V. Bertelsen, Distributing Real-Time Measurements of Speed Through Water from Ship to Shore, in: Proc. of 4th Hull Performance and Insight Conference, Gubbio, Italy, 2019.

[6] S. Barstow, J.-R. Bidlot, S. Caires, M. Donelan, W. Drennan, H. Dupuis, H. Graber, J. Green, O. Gronlie, C. Gurin, Measuring and Analysing the Directional Spectrum of Ocean Waves, COST Office, 2005

[7] M. Tucker, T. Pitt, Waves in Ocean Engineering, Vol. 5 of Elsevier Ocean Engineering Book Series, Elsevier, 2001.

[8] M. Tucker, Recommended standard for wave data sampling and near-real-time processing, Ocean Engineering 20 (1993) $459-474$.

[9] J. Nieto-Borge, K. Reichert, J. Dittmer, Use of nautical radar as a wave monitoring instrument, Coastal Engineering 37 (1999) $331-342$.

[10] U. Nielsen, A. Brodtkorb, A. Sørensen, Sea state estimation using multiple ships simultaneously as sailing wave buoys, Applied Ocean Research 83 (2019) 65-76.

[11] U. Nielsen, A concise account of techniques available for shipboard sea state estimation, Ocean Engineering 129 (2017) $352-362$.

[12] U. Nielsen, D. Stredulinksy, Sea state estimation from an advancing ship - A comparative study using sea trial data, Applied Ocean Research 34 (2012) 33-44. 
[13] F. de Souza, E. Tannuri, P. de Mello, G. Franzini, J. Mas-Soler, A. Simos, Bayesian Estimation of Directional Wave-Spectrum Using Vessel Motions and Wave-Probes: Proposal and Preliminary Experimental Validation, J. Offshore Mechanics and Arctic Eng. 140 (2018) 041102:110 .

[14] J. Mas-Soler, A. Simos, A Bayesian wave inference method accounting for nonlinearity related inaccuracies in motion RAOs, Applied Ocean Research (under review).

[15] A. Brodtkorb, U. Nielsen, A. Sørensen, Sea State Estimation Using Vessel Response in Dynamic Positioning, Applied Ocean Research 70 (2018) 76-86.

[16] U. Nielsen, A. Brodtkorb, A. Sørensen, A brute-force spectral approach for wave estimation using measured vessel motions, Marine Structures 60 (2018) 101-121.

[17] T. Iseki, K. Ohtsu, Bayesian estimation of directional wave spectra based on ship motions, Control Engineering Practice 8 (2000) $215-219$.

[18] E. A. Tannuri, J. V. Sparano, A. N. Simos, J. J. D. Cruz, Estimating directional wave spectrum based on stationary ship motion measurements, Applied Ocean Research 25 (2003) 243-261.

[19] U. Nielsen, Estimations of on-site directional wave spectra from measured ship responses, Marine Structures 19 (2006) 33-69.

[20] U. Nielsen, Introducing two hyperparameters in Bayesian estimation of wave spectra, Probabilistic Engineering Mechanics 23 (2008) 84-94.

[21] R. Bhattacharyya, Dynamics of Marine Vehicles, John Wiley \& Sons, 1978.

[22] R. Beck, W. Cummins, J. Dalzell, P. Mandel, W. Webster, Vol. III: Motions in Waves and Controllability, in: E. Lewis (Ed.), Principles of Naval Architecture, Second Revision, SNAME, 1989, pp. 1-188.

[23] G. Lindgren, I. Rychlik, M. Prevosto, Stochastic Doppler shift and encountered wave period distributions in Gaussian waves, Ocean Engineering 26 (1999) 507-518.

[24] U. Nielsen, Transformation of a wave energy spectrum from encounter to absolute domain when observing from an advancing ship, Applied Ocean Research 69 (2017) 160-172.

[25] U. Nielsen, Deriving the absolute wave spectrum from an encountered distribution of wave energy spectral densities, Ocean Engineering 165 (2018) 194-208.

[26] H. Akaike, Likelihood and Bayes Procedure, in: J. M. Bernado, M. H. D. Groot, D. U. Lindley, A. F. M. Smith (Eds.), Bayesian Statistics, University Press, Valencia, 1980, pp. 143-166.

[27] U. Nielsen, T. Iseki, Estimation of sea state parameters from measured ship responses The Bayesian approach with fixed hyperparameters, in: Proc. of 29th OMAE, ASME, Shanghai, China, 2010

[28] I. Da Silva Bispo, A. Simos, E. Tannuri, J. Da Cruz, Motion-based wave estimation by a bayesian inference method: A procedure for pre-defining the hyperparameters, in: Proc. of the International Offshore and Polar Engineering Conference, Rhodes, Greece, 2012.

[29] O. Phillips, The Dynamics of the Upper Ocean, 2nd ed., Cambridge University Press, 1977.

[30] J. Wolf, D. Prandle, Some observations of wave-current interactions, Coastal Engineering 37 (1999) 471-485.

[31] J. J. Jensen, Conditional Short-crested Waves in Shallow Water and With a Superimposed Current, in: Proc. of 21st OMAE, ASME, Oslo, Norway, 2002.

[32] N. Salvesen, E. O. Tuck, O. Faltinsen, Ship Motions and Sea Loads, Trans. SNAME 78 (1970) 250-287.

[33] U. Nielsen, Response-based estimation of sea state parameters - influence of filtering, Ocean Engineering 34 (2007) 1797-1810.

[34] U. Nielsen, The wave buoy analogy - estimating high-frequency wave excitations, Applied Ocean Research 30 (2008) 100-106.

[35] Y. Hong, T. Iseki, U. Nielsen, Short-term Variability of Cross-Spectral Analysis for Ship Responses in Waves, in: Proc. 17th Asia Navigation Conference, Chiba-city, Japan, 2018.

[36] Y. Hong, T. Iseki, U. Nielsen, The Effect of Short-term Variability of Cross-Spectral Analysis on Wave Buoy Analogy, in: Proc. 29th ISOPE, Honolulu, HI, USA, 2019.

[37] P. Hwang, Doppler frequency shift in ocean wave measurements: Frequency downshift of a fixed spectral wave number component by advection of wave orbital velocity, Journal Geophysical Research 111 (2006) C06033. 